\section{SCIENCE CHINA \\ Physics, Mechanics \& Astronomy}

\title{
Spintronic devices for neuromorphic computing
}

\author{
YaJun Zhang ${ }^{1}$, Qi Zheng ${ }^{1}$, XiaoRui Zhu ${ }^{1}$, Zhe Yuan ${ }^{1,2^{*}}$, and Ke Xia ${ }^{1,2,3 *}$ \\ ${ }^{1}$ Center for Advanced Quantum Studies and Department of Physics, Beijing Normal University, Beijing 100875, China; \\ 2 Peng Cheng Laboratory, Center for Quantum Computing, Shenzhen 518005, China; \\ ${ }^{3}$ Shenzhen Institute for Quantum Science and Engineering and Department of Physics, Southern University of Science and Technology, \\ Shenzhen 518055, China
}

Received November 28, 2019; accepted December 17, 2019; published online February 17, 2020 $\begin{array}{ll}\text { Citation: } & \text { Y. J. Zhang, Q. Zheng, X. R. Zhu, Z. Yuan, and K. Xia, Spintronic devices for neuromorphic computing, Sci. China-Phys. Mech. Astron. 63, } 277531 \\ & \text { (2020), https://doi.org/10.1007/s11433-019-1499-3 }\end{array}$

In the past decades, significant progress has been achieved in artificial intelligence, which is now widely applied in image recognition, big data analysis, unmanned vehicle control and other cognitive tasks [1]. These applications nevertheless are highly energy consuming partly because of the mismatch between the neural-network-computing-based software implementation and the von Neumann architecture of present computers. One promising solution is developing neuromorphic chips without the so-called von Neumann bottleneck, which are suitable for performing the desired computation based on artificial neural networks (ANNs). So far there have been demonstrations of neuromorphic chips using the traditional semiconductor techniques achieving very low energy consumption [2]. Such chips usually require thousands of transistors to simulate one spiking neuron and employ separate synapses from neurons. Therefore, many attempts are made to develop neuromorphic chips with new materials and technologies, e.g. resistive memristors [3], phase change materials [4], and ferroelectricity [5].

Spintronic devices, which were extensively studied as memory units, intrinsically have the required dynamical properties of the basic elements - neurons and synapses - in an ANN [6]. The nonlinear dynamics of an artificial neuron can be replaced by magnetization dynamics since the latter is described by the nonlinear Landau-Lifshitz-Gilbert equation.

*Corresponding authors (Zhe Yuan, email: zyuan@bnu.edu.cn Ke Xia, email: kexia@bnu.edu.cn)
The tunable resistance used in magnetic memory devices is naturally artificial synapses, whose nonvolatility further reduces the power consumption. In addition, the remarkably large endurance of magnetic devices $\left(>10^{15}\right)$ [7] is particularly suitable for implementing reprogrammable neural networks. Then the focus of the past spintronics research, which aimed to develop memory devices, shall be significantly extended to achieve spintronic neural networks. For instance, as a memory device, one only needs to probe the static states before and after magnetic switching while the dynamical process of magnetization is essential in the neuromorphic computing as well as the stochasticity.

A necessary step to realize the hardware of spintronic neural networks is finding the appropriate devices to replace the mathematical units in the computational models of artificial intelligence [8], where the neurons and synapses are usually described by differential equations. The synapses are the storage in neural networks and need to be tuned in the training process. Owing to the adjustable resistance, memristors are widely used in the implementation of hardware synapses [7]. The spintronic memristor can be designed by putting a magnetic domain wall on top of a collinear magnetic layer [9]. The resistance of this device is determined by the position of the domain wall, which can be continuously varied by an electrical current. MgO-based magnetic tunnel junction (MTJ) can also serve as a memristive device, where the resistance variation is controlled by the voltage-driven 
diffusion of oxygen atoms in $\mathrm{MgO}$ [10]. Another type of spintronic synapse consisting of $N$ MTJs that are serially connected can have up to $2^{N}$ discrete states of resistance [11]. Tuning the spintronic synapses following the algorithms of supervised and unsupervised learning rules has been experimentally demonstrated, including the long-term potentiation/depression and the more sophisticated spiking time dependent plasticity (STDP) [8]. Two classes of neurons are used in neuromorphic computing. One is commonly applied in conventional ANNs and a nonlinear activation function is used to mimic its dynamics [12], such as ReLU, Sigmoid, and a step function. The other type is used in the spiking neural networks inspired by the biological neurons. Mathematically it can be described by the leaky-integrate-firing (LIF) model. Such dynamics of the spiking neuron can be simulated by magnetization switching or domain wall motion: the magnetic response occurs only at the integrated stimulus above a certain threshold [13]. Some examples are schematically shown in Figure 1.

Many experimental studies try to improve these basic units of spintronic neural networks, but lack of criteria of neurons and synapses makes the technical route unclear towards the hardware with a better functionality. Even in the computational models, none of the nonlinear activation functions is clearly better than the others and many ANNs work equally well with different activation functions. An ultimate ver- (a)

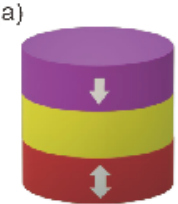

(d)

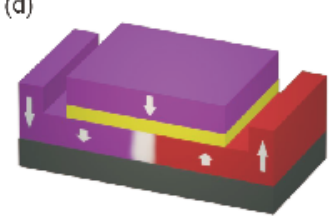

(f)

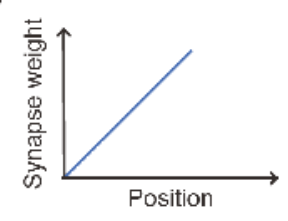

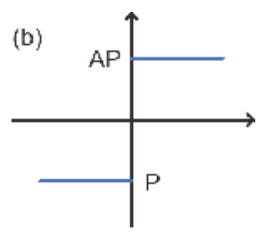

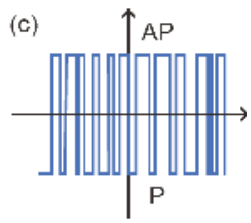

(e)

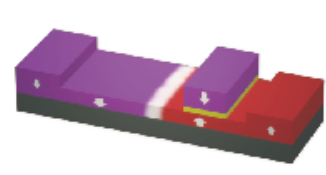

(g)

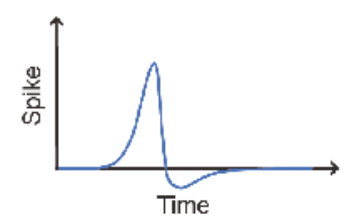

Figure 1 (Color online) (a) Sketch of a MTJ with a fixed (purple) magnetic layer and a free (red) layer. It has a low (high) resistance when the two layers have parallel (antiparallel) magnetization, as shown in (b). (c) In the superparamagnetic regime, the free layer is switched by thermal fluctuation and the resistance exhibits a random hopping between the two states. (d) Schematic illustration of a magnetic memristor. The free layer of a MTJ is replaced by a magnetic domain wall at the bottom, whose position determines the total resistance between the two magnetic layers (f). (e) A device to mimic a spike neuron. The firing occurs when the domain wall passes the electrode resulting in an abrupt change in the resistance $(\mathrm{g})$. ification can only be the performance of the constructed neural network using these artificial neurons and synapses.

There have been lots of proposals and attempts to implement neural networks using spintronic devices [14-16]. One example, a Hopfield-type ANN, is built using Hall bars as the synapses [14]. The Hall bars consist of a ferromagnetic $\mathrm{Co} / \mathrm{Ni}$ multilayer on top of antiferromagnetic PtMn, where the magnetization of $\mathrm{Co} / \mathrm{Ni}$ can be controlled by an electrical current via the so-called spin-orbit torque. Having a linear relationship between the Hall resistance and the writing current in the absence of an external magnetic field, the synaptic weights are easily tuned during the training. Integrated on a board, this Hopfield network is tested to memorize three letters, "I", "C", and "T" in $3 \times 3$ pixels. Limited by techniques and cost, more proposals are only based on simulation, where the device parameters can be obtained from either experiments or micromagnetics. For instance, crossbars are efficient devices to multiply a vector by a matrix, where spintronic devices like MTJs can be used to connect all the intersections, see Figure 2. The memristive properties of MTJs are exploited to mimic the adjustable synaptic weights. Simulations are performed to verify such crossbar-based ANNs, which are proposed to count number of vehicles [15] and to recognize handwritten numbers in the Mixed National Institute of Standards and Technology (MNIST) dataset [11]. In a more hierarchical simulation, a spiking neural network is proposed to be a crossbar connected via magnetic synapses [16], where the STDP can be applied for unsupervised learning. Combined with the CMOS-based LIF neurons, such a network can be utilized to recognize the handwritten digits in MNIST dataset. Nevertheless, these interesting proposals have yet to be examined in experiment, especially for the applicability of various training algorithms with the hardware synapses.

Recently, much attention is attracted by a special type of neuromorphic computing named reservoir computing, which is favorable for processing dynamical information. It is usually performed in a recurrent neural network (RNN) with a number of artificial neurons, which are sparsely and randomly connected. A RNN made of forty MTJs is trained to generate and recognize simple periodic functions [17], where
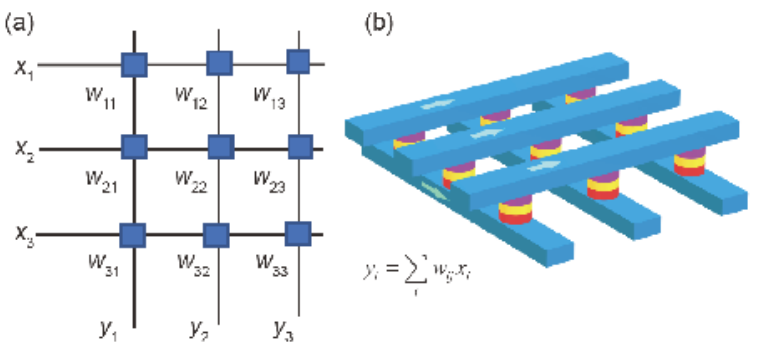

Figure 2 (Color online) (a) Sketch of a device to multiply a vector by a matrix; (b) a crossbar that can perform the multiplication, where the weights at connecting points correspond to the resistances of the MTJs. 
every neuron is implemented by a single MTJ. A systematic study shows that the RNN is robust against the phase noise of MTJs and imperfect synapses. Alternatively, a RNN can be implemented with a single nonlinear node that has a delayed feedback such as to reduce significantly the technical difficulty in experiment [18]. A single MTJ can remarkably improve the voice recognition owing to the nonlinear dynamics and memory effect [19]. Later, as demonstrated by a micromagnetic simulation, image recognition is achieved by using the dynamical motion of a single magnetic Skyrmion [20]. Because of its potential functionality and technical simplicity, the implementation of neuromorphic computing using a single spintronic device is likely to be applied for real cognitive tasks soon.

The conventional von Neumann computers require error correction to guarantee computational accuracy, in which the stochasticity must be suppressed with a remarkable energy cost. In a biological brain, random processes exist in the firing of neurons and signal transmission through synapses and play a key role in the complex cognitive and learning process. A stochastic neuron can be realized by a MTJ under a certain magnetic field and/or an electrical bias, where the energy barrier between the parallel and antiparallel configurations is comparable to $k_{\mathrm{B}} T$. Then the thermal fluctuation can flip the free-layer magnetization randomly in the socalled superparamagnetic regime. Such stochastic neurons are successfully applied in a Boltzmann machine and a Bayesian network [21] based on numerical simulations. The flipping rate of the MTJs is used to encode information in spiking neural networks [22].

The stochasticity is necessary in description of a quantum state and the random telegraphic switching of MTJs is applied in a circuit chip to perform integer factorization [23]. This probabilistic computing [24] opens an avenue of implementing quantum computing with spintronic devices. It is therefore possible to further develop a quantum neural network. Quantum reservoir computing is predicted to boost computational power by employing a quantum algorithm in the training process [25].

In summary, significant effort has been made in the emerging field of neuromorphic computing with spintronic devices. A few experimental studies have demonstrated the capability of spintronic neural networks for performing cognitive tasks and more proposals are suggested with multiscale simulations in combination with experiments. Neuromorphic computing can even be extended to apply quantum algorithms to develop quantum neural networks using the recently developed probabilistic computing. Since the information is encoded in the complex nonlinear dynamics, it is essential to study the whole network dynamics instead of the properties of its elements such as an artificial neuron and synapse. A further development of this field requires the indepth collaboration of computational neu- rosciences, physics, materials science, electronics, computer science, etc., where breaking the barriers down between different disciplines is crucial.

This work was supported by the National Natural Science Foundation of China (Grant No. 11734004).

1 Y. LeCun, Y. Bengio, and G. Hinton, Nature 521, 436 (2015).

2 M. Davies, N. Srinivasa, T. H. Lin, G. Chinya, Y. Cao, S. H. Choday, G. Dimou, P. Joshi, N. Imam, S. Jain, Y. Liao, C. K. Lin, A. Lines, R. Liu, D. Mathaikutty, S. McCoy, A. Paul, J. Tse, G. Venkataramanan, Y. H. Weng, A. Wild, Y. Yang, and H. Wang, IEEE Micro 38, 82 (2018).

3 P. Yao, H. Wu, B. Gao, S. B. Eryilmaz, X. Huang, W. Zhang, Q. Zhang, N. Deng, L. Shi, H. S. P. Wong, and H. Qian, Nat. Commun. 8, 15199 (2017); J. X. Shen, D. S. Shang, Y. S. Chai, S. G. Wang, B. G. Shen, and Y. Sun, Adv. Mater. 30, 1706717 (2018).

4 T. Tuma, A. Pantazi, M. Le Gallo, A. Sebastian, and E. Eleftheriou, Nat. Nanotech. 11, 693 (2016).

5 S. Boyn, J. Grollier, G. Lecerf, B. Xu, N. Locatelli, S. Fusil, S. Girod, C. Carrétéro, K. Garcia, S. Xavier, J. Tomas, L. Bellaiche, M. Bibes, A. Barthélémy, S. Saïghi, and V. Garcia, Nat. Commun. 8, 14736 (2017).

6 J. Grollier, D. Querlioz, and M. D. Stiles, Proc. IEEE 104, 2024 (2016).

7 J. J. Yang, D. B. Strukov, and D. R. Stewart, Nat. Nanotech. 8, 13 (2013).

8 Y. Cao, A. W. Rushforth, Y. Sheng, H. Zheng, and K. Wang, Adv. Funct. Mater. 29, 1808104 (2019).

9 X. B. Wang, Y. R. Chen, H. W. Xi, H. Li, and D. Dimitrov, IEEE Electron Device Lett. 30, 294 (2009).

10 P. Krzysteczko, J. Münchenberger, M. Schäfers, G. Reiss, and A. Thomas, Adv. Mater. 24, 762 (2012).

11 D. Zhang, L. Zeng, K. Cao, M. Wang, S. Peng, Y. Zhang, Y. Zhang, J. O. Klein, Y. Wang, and W. Zhao, IEEE Trans. Biomed. Circuits Syst. 10, 828 (2016).

12 J. L. Cai, B. Fang, L. K. Zhang, W. X. Lv, B. S. Zhang, T. J. Zhou, G. Finocchio, and Z. M. Zeng, Phys. Rev. Appl. 11, 034015 (2019).

13 A. Kurenkov, S. DuttaGupta, C. Zhang, S. Fukami, Y. Horio, and H. Ohno, Adv. Mater. 31, 1900636 (2019).

14 W. A. Borders, H. Akima, S. Fukami, S. Moriya, S. Kurihara, Y. Horio, S. Sato, and H. Ohno, Appl. Phys. Express 10, 013007 (2017).

15 A. F. Vincent, J. Larroque, N. Locatelli, N. Ben Romdhane, O. Bichler, C. Gamrat, W. S. Zhao, J. O. Klein, S. Galdin-Retailleau, and D. Querlioz, IEEE Trans. Biomed. Circuits Syst. 9, 166 (2015).

16 A. Sengupta, A. Banerjee, and K. Roy, Phys. Rev. Appl. 6, 064003 (2016).

17 Q. Zheng, X. Zhu, Y. Mi, Z. Yuan, and K. Xia, AIP Adv. 10, 025116 (2020). arXiv:1912.08596.

18 L. Appeltant, M. C. Soriano, G. van der Sande, J. Danckaert, S. Massar, J. Dambre, B. Schrauwen, C. R. Mirasso, and I. Fischer, Nat. Commun. 2, 468 (2011).

19 J. Torrejon, M. Riou, F. A. Araujo, S. Tsunegi, G. Khalsa, D. Querlioz, P. Bortolotti, V. Cros, K. Yakushiji, A. Fukushima, H. Kubota, S. Yuasa, M. D. Stiles, and J. Grollier, Nature 547, 428 (2017).

20 W. Jiang, L. Chen, K. Zhou, L. Li, Q. Fu, Y. Du, and R. H. Liu, Appl. Phys. Lett. 115, 192403 (2019).

21 Y. Shim, S. Chen, A. Sengupta, and K. Roy, Sci. Rep. 7, 14101 (2017).

22 A. Mizrahi, T. Hirtzlin, A. Fukushima, H. Kubota, S. Yuasa, J. Grollier, and D. Querlioz, Nat. Commun. 9, 1533 (2018).

23 W. A. Borders, A. Z. Pervaiz, S. Fukami, K. Y. Camsari, H. Ohno, and S. Datta, Nature 573, 390 (2019).

24 K. Y. Camsari, B. M. Sutton, and S. Datta, Appl. Phys. Rev. 6, 011305 (2019).

25 K. Nakajima, K. Fujii, M. Negoro, K. Mitarai, and M. Kitagawa, Phys. Rev. Appl. 11, 034021 (2019). 